\title{
Inhibition of Mitochondrial Fission by Drp-1 Blockade Improves White Adipose Tissue Abnormalities in Obesity and Diabetes
}

\author{
Paola Finocchietto ${ }^{1}$, Hernan Perez ${ }^{1}$, Guillermo Blanco ${ }^{2}$, Verónica Miksztowicz ${ }^{2}$, Clarisa \\ Marotte $^{1}$, Celina Morales ${ }^{3}$, Jorge Peralta ${ }^{1}$, Gabriela Berg ${ }^{2}$, Cecilia Poderoso ${ }^{4}$, Juan Jose \\ Poderoso $^{1}$, and Maria Cecilia Carreras ${ }^{1}$ \\ ${ }^{1}$ Hospital de Clinicas Jose de San Martin \\ ${ }^{2}$ Facultad de Farmacia y Bioquímica UBA \\ ${ }^{3} 3$ Institute of Cardiovascular Physiopathology, School of Medicine, University of Buenos \\ Aires \\ ${ }^{4}$ UBA - Facultad de Medicina
}

July 27, 2020

\begin{abstract}
Background and Purpose: Obesity and Type 2 Diabetes are major causes of morbidity-mortality characterized by mitochondrial dysfunction and oxidative-nitrosative stress. Despite intensive research, the events that cause the onset and progression of these diseases are not completely understood. Herein, we investigated if dysregulation of mitochondrial dynamics and biogenesis is involved in an animal model of obesity and diabetes. Experimental Approach: Mitochondrial dynamics and biogenesis were evaluated in epididymal white adipose tissue and adipocytes from ob/ob mice, an animal model of obesity and diabetes, pharmacological treatment with mdvi-1, a selective inhibitor of Drp1 and leptin. Key Results: A decrease of Mfn2 and OPA-1 protein expression and an increase in Drp1- protein levels were observed with enhanced and sustained mitochondrial fragmentation in ob/ob mice compared to wt C57BL/6 animals. The content of mitochondrial DNA and mRNA expression of PGC- $1 \alpha$, both parameters of mitochondrial biogenesis, were reduced in ob/ob mice. The treatment with leptin or mdvi-1 (Drp1 inhibitor) significantly increased abnormal biogenesis, improved fusion-to-fission balance and attenuated mitochondrial dysfunction, and adipogenesis, thus inducing white-to-beige adipocyte transdifferentiation. Measurements of glucose and lipid oxidation in adipocytes revealed that both leptin and mdvi-1 increase substrates oxidation while in vivo determination of blood glucose showed decreased blood glucose concentration by $50 \%$ in ob/ob mice, almost to the wt level. Conclusion and Implications: In light of these results, pharmacological targeting of Drp1 may be a potential novel therapeutic tool for obesity and diabetes.
\end{abstract}

Abbreviations Used

AMPK: AMP-dependent kinase

BAT brown adipose tissue

DAF-FM-amino-5-methylamino-2థ,7\$-difluorofluorescein diacetate

DRP-1 dynamin-related protein-1

FFA free fatty acid

LC3 Microtubule-associated protein 1A/1B-light chain 3

Mdvi-1 selective inhibitor of Drp1 
Mfn2 mitofusin proteins

NO nitric oxide

NRF1 nuclear respiratory factor

$\mathrm{Ob} / \mathrm{ob}$ Leptin-deficient mice

OPA-1 optic atrophy-1

OXPHOS: oxidative phosphorylation system

PGC-1 $\alpha$ proliferator-activated receptor gamma coactivator-1 alpha

RNS reactive nitrosative species

ROS reactive oxygen species

siRNA small interference RNA

UCP-1 uncoupler protein 1

WAT white adipose tissue

\section{INTRODUCTION}

Obesity and Type 2 Diabetes are chronic diseases that typically coexist and have grown in prevalence to become a global health problem. This close relationship led to coining of the term 'diabesity', highlighting the fact that the two diseases share a pathophysiological axis of insulin resistance (IR), oxidative stress, mitochondrial dysfunction and chronic inflammation (Leitner et al., 2017; Boden, 2011).

Diabesity is characterized by an abnormal increase in white adipose tissue (WAT) mass with adipocyte hypertrophy and hyperplasia, depicting an imbalance between energy intake and consumption that leads to energy overload (Hildebran et al., 2018). In mammals, there are three types of adipose tissues: white, brown and beige. These subtypes have distinct functions and morphology, different protein expression patterns, and dissimilar developmental origins (Pfeifer et al., 2015). While the function of WAT is to store energy in the form of lipid droplets, that can be released to fuel other tissues, brown adipose tissue (BAT) has thermogenic properties for maintaining body temperature. Beige or brite (brown-in-white) fat is predominantly found interspersed in WAT depots and can acquire a brown-like phenotype upon cold exposure or pharmacological stimulation 1 (UCP1) that enables the rapid generation of heat and the oxidation of lipids or glucose. In contrast to WAT, there is an abundance of

Mitochondria both in brown and beige adipocytes, which unlike other tissues such as liver or skeletal muscle, are enriched in mitochondrial uncoupling proteins playing a role in systemic glucose homeostasis (Chen et al., 2016).

Mitochondria are dynamic organelles essential for cellular energy survival whose morphology has a remarkable plasticity, undergoing constant mitochondrial fusion and fission, which are necessary for the quality control process (Chandel, 2014). As recently published, mitochondrial quality control and interaction with the nucleus are critical for the maintenance of their function (Kotiadis et al., 2014). Energetic homeostasis is sustained through the balance among mitochondrial biogenesis, dynamics, ultrastructure, function and degradation in a healthy mitochondrial population. Mitochondrial fusion is mediated by the mitofusin proteins (Mfn) 1 and 2 in the outer membrane and by OPA-1 (optic atrophy-1) in the inner membrane. Mitochondrial fusion dilutes the oxidative stress damaged lipids, proteins and mitochondrial DNA, enabling repolarization of the membrane and retaining bioenergetic capacity. Mitochondrial fission allows damaged mitochondria to be removed by mitophagy which is a selective form of autophagy whereby mitochondria are selectively degraded and biosynthetic blocks are recycled (Westermann et al., 2010). Mitochondrial fission requires the translocation of Drp-1, a member of the large GTPases dynamic family, from the cytosol to the mitochondrial outer membrane. Post-translational modifications of the protein, mainly, by phosphorylation 
of serine residues that increase or decrease its GTPase activity, contribute to the regulation of mitochondrial fission (Mootha et al.,2011). While phosphorylation of Drp1 on Ser-637prevents its mitochondrial translocation phosphorylation of Drp1 on Ser-616 promotes mitochondrial fragmentation during mitosis. Under hyperglycaemic conditions, O-GlcNAcylation of OPA1 and Drp1 causes dephosphorylation on Ser-637 and the translocation of Drp1 to the outer mitochondria membrane (Chen et al., 2005; Yu et al., 2006). Pursuant to available basic approaches aimed at impairing Drp1 function in cells, mitochondrial fission can be prevented by pharmacological inhibition with Mdivi-1, a quinazolinone that selectively inhibits Drp1 over other dynamin family members. This approach gains ascendency considering that short-term use of mdivi-1 has been shown to be safe (Smith et al., 2017).

Mitochondrial biogenesis, the process through which new mitochondria are generated is different from mitochondrial fission because it involves the complete replication of mitochondrial DNA, which is particularly susceptible to oxidative damage due to its naked structure and proximity to the free radical production site.

Mitochondrial DNA damage results in incomplete synthesis of respiratory chain proteins and exacerbation of reactive oxygen species (ROS) generation. Mitochondrial biogenesis is driven by the transcriptional activators NRF-1 and 2 and by PGC-1 alpha, which is activated by various signaling pathways, including nitric oxide (NO)/cyclic GMP, Akt and AMPK among others (Whitaker et al., 2016). The AMP-activated protein kinase (AMPK) is a cellular energy sensor that when activated by phosphorylation drives to upregulation of PGC1 $\alpha$, UCP1, and mitochondrial biogenesis (Desjardins et al., 2018).

The predominance of fission leads to mitochondrial fragmentation, while an excess in fusion leads to the tubular elongation of mitochondria (Baker et al.2014). Alterations in the fission-/-fusion ratio produce morphological abnormalities and impair multiple mitochondrial functions, such as respiration, ATP synthesis and cell proliferation. The involved mechanisms are related to increased ROS production, oxidative and nitrosative stress, decreased membrane potential, mitochondrial DNA loss, and activation of autophagy.

Alterations of mitochondrial dynamics and function have been implicated in neurodegeneration, aging, sepsis, cardiovascular disease, obesity and type 2 diabetes. In patients with obesity or type 2 diabetes, alterations in mitochondrial fusion associated with reduced Mfn-2 expression and small-sized fragmented and swollen mitochondria, have been observed in different cell types, including liver, heart and pancreas (Archer, 2013; Perez et al., 2018; Gonzalez et al., 2014; Rovira-Llopis et al., 2017). Likewise, our group reported the presence of mitochondrial dysfunction, with subsequent disruption of energy metabolism and oxidative/nitrosative stress affecting mitochondrial complex I activity in WAT, liver and skeletal muscle from $o b / o b m i c e$ (Finocchietto et al., 2008; Finocchietto et al., 2011). These rodents constitute a well-known model of obesity and type 2 diabetes, since $o b / o b$ mice suffer a mutation that determines a premature stop codon in the Lep gene (leptin). This genetic condition leads to the transcription of a short mRNA variant and the formation of inactive leptin (Pelleymounter et al., 1995). Leptin is an adipokine released from white fat tissue that acts on the hypothalamus to induce satiety and reduce food intake, and, on the peripheral tissues to control metabolism and to increase the metabolic rate. Although genetic leptin deficiency is rare in human beings, resistance to leptin is a prominent feature of obese subjects with hypometabolism. In this context, leptin deficiency is associated to a definite phenotype including obesity, high food intake, decreased mitochondrial respiration, poor energy expenditure and fat accumulation (Ceddia, 2005).

In this context, the twofold aim of the present study is to investigate if dysregulation of mitochondrial dynamics and biogenesis is involved in a clinically relevant animal model of obesity and diabetes, and if Drp1 can be pharmacologically targeted as a potential novel therapeutic tool for obesity and diabetes.

\section{MATERIALS AND METHODS}

\section{Animals}

Male $o b / o b( \pm 70 \mathrm{~g})$ and $w t C 57 B L / 6$ ( $\pm 25 \mathrm{~g}) 5$ months old mice were purchased from Jackson Labs. Animal experiments were performed in accordance with the Principles of Laboratory Animal Care. The animal experiments were approved by the local Scientific and Technology Ethics Committee at the University 
of Buenos Aires (UBA). All efforts were made to minimize animal suffering and to reduce the number of animals used. Mice were maintained under controlled temperature $\left(22^{\mathrm{O}} \mathrm{C}\right)$, humidity $(50-60 \%)$, and airflow conditions, with a fixed 12-h light/dark cycle. Until the experiment onset, all animals were fed with a standard rat laboratory chow and had free access to food and water to standardize their nutritional status. Animals were sacrificed by cervical dislocation at the end of the treatment with leptin or mdvi- 1 .

\section{Experimental animals and drug administration}

During 3 days, Wt $C 57 \mathrm{BL} / 6$ and $\mathrm{Ob} / \mathrm{ob}$ mice received intraperitoneal injection of pyrogen-free dimethylsulfoxide or recombinant leptin (1 mg/kg/d dissolved in dimethyl-sulfoxide) (L 3772 Sigma Chemical Co.), or mdvi-1 (50 mg/kg/d dissolved in dimethyl-sulfoxide) (Mdivi-1 MO199 Sigma Chemical Co). In all mice, body weight and food intake were measured.

\section{Blood glucose concentration}

On the day of sacrifice, blood samples were collected using cardiac puncture. Blood glucose concentration was determined by Accu-check performance glucometer (Roche Lab).

\section{White adipose tissue extraction}

Mice were sacrificed by cervical dislocation and epididymal white adipose tissue (representative of visceral adipose tissue) was immediately extracted and homogenized in sucrose buffer 20\% (TRIS $10 \mathrm{mM}$, EDTA $0.1 \mathrm{mM}$, sucrose $20 \%, 2 \%$ protease inhibitor cocktail (Sigma Aldrich, St. Louis, MO, USA). The homogenate was centrifuged at $800 \mathrm{~g}$ for $10 \mathrm{~min}$ at $4^{\circ} \mathrm{C}$, frozen in liquid nitrogen and stored at $80^{\circ} \mathrm{C}$ until further analysis.

\section{Immunoblotting}

Proteins $(50 \mu \mathrm{g})$ were separated using electrophoresis on 7.5-10\% SDS-polyacrylamide gel, and transferred to a PVDF membrane (GE, Healthcare). Membranes were incubated with antibodies against 1: 1000 antiMfn2 (H-68): sc-50331, 1: 4000 anti-actin (I- 19): sc-1616 obtained from Santa Cruz, CA or 1: 1000 anti-OPA1: 612607 and 1: 1000 anti-Drp1: 611113 obtained from BD Biosciences or 1: 1000 anti-phosphoDrp1 (Ser616): \#3455, 1: 1000 anti-phospho-Drp1 (Ser637): \#6319, 1:1000 anti-AMPKa: \#2532, 1:1000 anti-phospho AMPK $\alpha$ (Thr172): \#2531 obtained from Cell Signaling. After several washes, the membranes were incubated with appropriate horseradish peroxidase conjugated secondary antibodies. Detection of immunoreactive proteins was accomplished by chemiluminescence with ECL. Quantification of bands was performed by digital image analysis using Totallab analyzer software (Nonlinear Dynamics Ltd, Biodynamics, Argentina). Equal loading was controlled with the appropriate markers. Isolation of white adipose tissue mitochondria

WAT mitochondria were isolated from homogenized tissue by differential centrifugation. Mitochondrial pellets were stored in the presence of antiproteases and antiphosphatases as described (Finocchietto et al., 2008; Finocchietto et al., 2011).

\section{Electron microscopy}

White adipose tissue was fixed in $4 \%$ paraformaldehyde, $2 \%$ glutaraldehyde, and $5 \%$ sucrose in PBS, followed by 2 -h post-fixation in $1 \%$ osmium tetroxide, and then $1 \mathrm{~h}$ in uranyl acetate in $50 \%$ ethanol. Samples were washed with ethanol $50 \%$ and dehydrated with a graded series of ethanol, clarified with acetone and embedded in Vestopal. Grids were prepared and stained with uranyl acetate and lead citrate. Samples were observed at $100 \mathrm{kV}$ with a Zeiss EM-109-T transmission electron microscope (Zeiss, Oberkochen, Germany).

\section{Primary fat cell isolation}

Fat pads were weighed and sliced into $3 \mathrm{~mm}$ pieces with scissors and resuspended in $5 \mathrm{ml}$ of KRH buffer $(25$

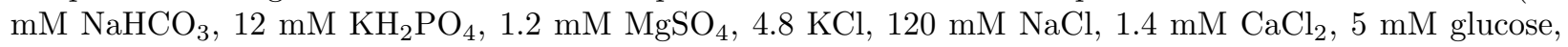
$20 \mathrm{mM}$ Hepes, $2 \%$ protease inhibitor cocktail (Sigma Aldrich, St. Louis, MO, USA), pH7.4, plus 2.5\% BSA containing $0.5 \mathrm{mg} / \mathrm{ml}$ collagenase (Sigma Chemical Co., St Louis, MO). The fat pads were digested for 45-60 minutes at $37^{\circ} \mathrm{C}$ in an orbital bath shaking at $100 \mathrm{rpm}$. Adipocytes released from the tissue were harvested 
by centrifugation (400g, $15 \mathrm{~min}$ ). Cells in the upper phase of the centrifuge tube were collected, washed three times and resuspended in $0.15 \mathrm{M}$ phosphate-buffered saline.

Adipocytes were isolated from animals without treatment and under the different treatments.

\section{Fluorescence microscopy}

Adipocytes from mice under treatment were stained with mitotracker green (50uM) (Invitrogen Carlsbad, CA) and observed under an Olympus BX-51 fluorescence microscope equipped with a digital Q-Color 3 Olympus camera. Digital images were processed with Image-Pro Plus 6.0 software (Media Cybernetics, Rockville, MD, USA) and Cell Profiler (Broad Institute, USA). A Cell Profiler pipeline was created for the segmentation of individual mitochondria and measurement of morphometric parameters. Several images for each treatment were processed with this pipeline to identify and measure more than 300 mitochondria. All morphometric measurements of mitochondria were exported to a standard database and were further analysed with Cell Profiler Analyst (Broad Institute, USA). A standard random forest classification algorithm was used to implement a machine learning approach to classify mitochondria intro three groups: round, elongated and branched. The training procedure was repeated until global classification accuracy achieved $90 \%$, with $100 \%$ classification accuracy of the round mitochondria subgroup. Then the whole set was scored for the amount of mitochondria belonging to each of the three groups (Carpenter et al., 2006; Jones et al., 2008; Jones et al., 2009).

\section{Optic microscopy and histological evaluation}

The histological examination by light microscopy was performed in a blinded manner. Fixed epididymal WAT samples were dehydrated in ethanol, embedded in paraffin wax, and cut with a microtome Reichert (Austria). The resulting micro-sections were stained with hematoxylin and eosin reagent and periodic acidschiff (PAS) stain for the determination of size and density of adipocytes and vascular density. In both cases the quantification was performed at high power field (HPF), 20 fields at 400x magnification for each animal using a computerized image analyzer (Image Pro Plus, Media Cybernetics Corp).

\section{Quantitative real-time PCR (RT-qPCR)}

Total RNA was insulated from the different tissues using TriZol reagent following the manufacturer's instructions (Life Technologies, Inc.-BRL, Grand Island, NY). Any residual genomic DNA was removed by treating RNA with RQ1 Rnase-free DNase (Promega, Madison, WI, USA) at $37^{\circ} \mathrm{C}$ for $30 \mathrm{~min}$, which was subsequently inactivated by incubation with $2 \mathrm{mM}$ EGTA for $10 \mathrm{~min}$ at $65^{\circ} \mathrm{C}$. First-strand cDNA synthesis was performed using oligo(dT)18 primer, Invitrogen SuperScript IV Reverse Transcriptase (RT), and RNase inhibitor (RNasin, Promega, Madison, WI, USA). PCR amplification and analysis were performed with ABI PRISM 7500 Sequence Detector System (PE Applied Biosystems, Foster City, CA). SYBR Select Master Mix (Applied Biosystems, Carlsbad, CA, USA) was used for all reactions, following manufacturer's instructions. Real-time PCR data were analyzed by calculating the $2^{-\Delta \Delta^{\prime \prime} \tau}$ value (comparative Ct method) using GAPDH expression as housekeeping, performed in parallel as endogenous control. The primer sequences and the condition of each reaction are shown in Supplemental Table I.

\section{mtDNA/nDNA ratio}

Both WAT or liver tissue were homogenized in lysis buffer $(10 \mathrm{mM}$ Tris-HCl pH 8, $1 \mathrm{mM}$ EDTA, and $0.1 \%$ SDS). After adding proteinase $\mathrm{K}$, lysates were incubated at $55{ }^{\circ} \mathrm{C}$ for $3 \mathrm{~h}$, vigorously vortexed and centrifuged (8,000 $\mathrm{g}$ for $15 \mathrm{~min})$; the resting supernatant was vortexed with $1 \mathrm{ml}$ protein precipitation solution (Gentra Puregene Kit) for $30 \mathrm{sec}$ and placed on ice for $5 \mathrm{~min}$. The resulting supernatant was mixed with 1 volume of isopropanol and centrifuged $\left(12,000 \mathrm{~g}\right.$ for $15 \mathrm{~min}$ at $\left.4{ }^{\circ} \mathrm{C}\right)$ to precipitate DNA. The DNA pellets were washed with $70 \%$ ethanol, air dried, and dissolved in Tris-EDTA buffer. For mtDNA/nDNA qPCRs, 40 ng of total genomic DNA were used. The mtDNA/nDNA ratio was calculated using the formula: $\left.2 \times 2^{\left(\Delta^{\prime \prime}\right.}{ }_{\mathrm{T}}\right)$, where $\Delta \mathrm{C}_{\mathrm{T}}$ is the difference of $\mathrm{C}_{\mathrm{T}}$ values between nDNA $32 \mathrm{M}$ and the mtDNA $16 \mathrm{~S}$ rRNA primers as shown in (Venegas el al.,2011). 


\section{Mitochondrial enzyme activity}

The activity of Complex I (NADH: ubiquinone reductase) was determined spectrophotometrically following the reduction of $50 \mu \mathrm{M}$ 2,3-dimethoxy-6-methyl-1,4-benzoquinone at $340 \mathrm{~nm}$ by $50 \mu \mathrm{g} / \mathrm{ml}$ mitochondrial proteins with a Hitachi U3000 spectrophotometer at $30^{\circ} \mathrm{C} ; 200 \mu \mathrm{M}$ NADH was used as electron donor. Reaction was carried out in the presence of $1 \mathrm{mM} \mathrm{KCN}$ and expressed as nmol of reduced benzoquinone/min.mg prot. Complex I activity was selective inhibited by $10 \mu \mathrm{M}$ rotenone.

\section{In vitro white adipose cells SiRNA transfection}

Adipocytes were transfected with siRNAsDrp-1 or control siRNA (Santa Cruz Biotechnology) using lipofectamine (Invitrogen Corp. California, USA) in Opti-MEM reduced serum medium and were incubated at $37^{\circ} \mathrm{C}$ in a $\mathrm{CO} 2$ for 10 hours, according to the protocol provided by the manufacturer. Silencing efficiency was evaluated by Drp-1 immunoblotting. The sequence of siRNAs Drp-1-sc-45953 was designed on the structure of mice genome.

\section{Fatty acid metabolism}

Adipocytes isolated were incubated with leptin (200 ng/ml), mdvi-1 (50 uM) or siRNA Drp-1 $2.5 \mathrm{ug} / \mathrm{ul}$ for $10 \mathrm{~h}$, and with $[14 \mathrm{H}]$ palmitic acid, samples were distributed into tubes containing Whatman filter paper soaked in $\mathrm{NaOH}$ and $200 \mathrm{nCi} / \mathrm{ml}[14 \mathrm{C}]$ palmitic acid. The tubes were sealed and incubated for $2 \mathrm{~h}$; then, 10 $\mathrm{N} \mathrm{HCl}$ was added to release $[14 \mathrm{C}] \mathrm{CO}$, which was detected by scintillation counting of the filter paper. To measure complete glucose oxidation to $\mathrm{CO}_{2}$ and $\mathrm{H}_{2} \mathrm{O}$, samples were supplemented with D-glucose [14C[U]][250 mCi/mmol]. Radioisotopes were from Perkin-Elmer Life and Analytical Sciences, Boston, MA, USA.

\section{Statistical analysis}

Data are presented as mean +- SEM according to the normal or skewed distribution. Significant differences between groups were accepted at $\mathrm{p}<0.05$. One-way ANOVA (multigroup comparisons) followed by Bonferroni's multiple comparison test or Dunnett's test were carried out with GraphPad Prism 5.01 (La Jolla, CA).

\section{RESULTS}

\section{Mitochondrial dynamics is abnormal in visceral WAT fromob/ob mice}

In order to determine whether mitochondrial dynamics is impaired in leptin-deficient $o b / o b$ mice, we analyzed the expression of the mitochondrial fission protein Drp1 and its activation status. We found that Drp1 protein level is increased by $\sim 50 \%$ in the WAT of $o b / o b$ mice compared to wild type animals (Fig. 1A). Drp1 activity is regulated by the opposing effects of phosphorylation at two key serine residues: phosphorylation of Ser 616 increases Drp1 activity whereas phosphorylation of Ser 637 decreases it (Otera et al., 2013).

We measured the phosphorylation of both residues and found an increase in p-Ser 616-Drp1 (active form) whereas p-Ser 637-Drp1 (inactive form) decreased in $o b / o b$ mice (Fig. $1 \mathrm{~B}$ and C). Importantly, administration of leptin reverted the changes in Drp1 expression and phosphorylation in $o b / o b$ mice stimulating the phosphorylation of p-Ser 637-Drp1 (Figs. 1 A-C). In addition, we observed a reduction in Mfn2 and OPA1 protein levels (mitochondrial fusion proteins) inob/ob, which was restored after leptin treatment (Fig. $1 \mathrm{D}$ and $\mathrm{E})$. These results suggest an imbalanced mitochondrial dynamic in visceral white adipose tissue of leptin-deficient mice. No changes were observed in wt C57BL/6 under any treatments.

\section{Drp-1 blockade reverts mitochondrial morphological changes in WAT from $o b / o b$ mice}

After observing an overexpression of Drp-1 protein in $o b / o b$ mice, TEM was used to detect mitochondrial morphology defects in $o b / o b$ mice WAT slices in comparison to those from $w t C 57 B L / 6$ mice. While $w t$ mitochondria were predominantly tubular-shaped $(80 \%), o b / o b$ mitochondria were aberrantly small and spherical in shape (85\%) (Fig. $2 \mathrm{~A}$ and B). Short treatment of $o b / o b$ mice with leptin or mdvi-1 resulted in a significant increase in tubular-shaped mitochondria ( $55 \%$ and $49 \%$, respectively) (Fig. 2 C, D and E), suggesting that 
the small, spherical and fragmented mitochondria are characteristic of $o b / o b$ mice WAT a result of increased mitochondrial fission.

Mitochondria are replaced every 2-4 weeks in different tissues (Twig et al., 2011). The destruction of mitochondria is believed to occur through mitophagy, an organelle-specific form of autophagy, a process in which parts of the cytoplasm, including organelles, are sequestered in double-membrane autophagic vacuoles or autophagosomes, as a cell-survival mechanism. Mitophagy is essential for mitochondrial quality control and regulation of mitochondrial mass. Taking into account that mitochondrial fragmentation and dysfunction lead to mitophagy (Navarro-Yepes et al., 2014), we analyzed WAT slices by TEM and observed mitochondria engulfed by double-membrane vacuoles with mitochondrial fragments, indicating an active process of mitophagy in $o b / o b$ mice (10 images per field). These alterations were reduced after treatment with leptin ( 5 images per fields) or mdvi-1 (3 images per field) (Fig. 2F). No changes were observed in wt $C 57 B L / 6$ under any treatments.

\section{Drp-1 blockade reverts mitochondrial morphological changes in isolated adipocytes}

In order to investigate the effects of Drp-1 inhibition on mitochondria morphology caused by leptin and mdvi1, we studied culture and isolated adipocytes from $w t C 57 B L / 6$ or $o b / o b$ mice treated with leptin or mdvi-1 intraperitoneally. Mitochondrial shape changes can be informative indicators of the mitochondrial network bioenergetics performance (Galloway et al., 2012). We investigated mitochondria morphological changes using a machine learning algorithm to allow automated classification of mitochondria shape into three groups: branched, round, or elongated detected by fluorescence microscopy images (Suppl. Fig. 1 A). "Branched" mitochondria denoted complex shapes including T-shapes, Y-shapes, P-shapes, O-shapes, among others, and have been associated with particular bioenergetic profiles. The results of mitochondrial classification into each of the three groups through machine learning is shown in Fig. $3 \mathrm{~A}$ and $\mathrm{B}$. The measurement confirmed that $o b / o b$ mice had a higher proportion of round mitochondria compared to $w t C 57 B L / 6$ groups $(0.45+-0.05$ to $0.22+-0.04$ ), consistent with the TEM analysis in WAT slices. This proportion was partially restored in $o b / o b$ mice treated with leptin $(0.36+-0.04)$, and completely restored or even decreased by mdvi- 1 treatment $(0.22+-0.05)$. The proportion of elongated mitochondria was strongly reduced in $o b / o b$ mice when compared against wt $C 57 B L / 6$ mice $(0.19+-0.04$ and $0.47+-0.06$, respectively). Both leptin and mdvi-1 treatment increased the presence of elongated mitochondria $(0.31+-0.04$ and $0.31+-0.06$, respectively). In contrast, $w t$ $C 57 B L / 6, o b / o b$ and leptin-treated groups showed a similar proportion of branched mitochondria (0.31+$0.06,0.36+-0.05$ and $0.32+-0.06$, respectively), while mdvi-1-treated mice showed an increased proportion of branched mitochondria (0.46+-0.05) (Fig. 3B and Suppl. Fig. 1). No changes were found in $w t C 57 B L / 6$ under any treatment.

\section{AMPK and mitochondrial biogenesis are reduced in WAT fromob/ob mice}

AMPK is a key regulator of energy metabolism and a central trigger of mitochondrial biogenesis through activation of PGC-1 $\alpha$ and downstream nuclear factors (Jeon, 2016). We previously reported the existence of a mitochondrial pool of active (phosphorylated) AMPK in normal adipocytes (Finocchietto et al.,2011). Investigation of AMPK phosphorylation inob/ob homogenates revealed that AMPK was completely devoid in its phosphorylated form (active) and increased with leptin or mdvi-1 treatment (Fig. 4 A and B).

PGC- $1 \alpha$ plays a critical role in controlling mitochondrial biogenesis under AMPK regulation (Jager et al., 2007). Obesity and diabetes are associated with impairment of PGC- $1 \alpha$ signaling and defects in mitochondrial biogenesis (Zorzano et al., 2009). To determine the effect of leptin or Mdvi-1 treatment in mitochondrial biogenesis of $o b / o b$ mice WAT, we assessed PGC-1 $\alpha$ gen expression through q PCR. The mRNA level of PGC-1 $\alpha$ was significantly lower in $o b / o b$ mice compared to wt $C 57 B L / 6$, leptin or mdvi-1 treatment (Fig 4 C).

In agreement with the reduced mRNA PCG-l $\alpha$ level, the relative mass of mitochondria, assessed by mtDNA content, showed a strong reduction inob/ob mice compared to wt C57BL/6 . Remarkably, inhibition of Drp-1 by mdvi-1 led to increased mtDNA content indistinguishable from leptin (Fig. $4 \mathrm{D}$ ). This finding suggests that enhanced mitochondrial fission and mitophagy with a decrease in biogenesis underlie the loss 
of mitochondria in $o b / o b$ mice.

\section{Drp-1 blockade induces white-to-beige adipocyte transdifferentiation}

Brown adipocytes regulate energy expenditure through their numerous, large mitochondria. The brown adipose tissue (BAT)-specific uncoupling protein 1 (UCP-1) is localized in the inner mitochondrial membrane, and when activated, it dissipates the intermembrane proton-gradient and generates heat instead of ATP. White adipocytes can be reprogrammed and transdifferentiated into brown-like adipose tissue or beige adipocytes. Brown and beige adipocyte share many properties, including multilocular lipid droplets, less adipocyte area, vascular density, dense mitochondria and expression of UCP (Liangyou, 2018).

In order to study whether the treatments with leptin or mdvi-1 inob/ob mice stimulate the "browning" process in visceral white adipose tissue, we measured the below mentioned parameters and observed a decrease in the adipocyte area and an increase in the vascular density (Fig. 5 A, B and C). Surprisingly mRNA UCP-1 expression was increased in AT with leptin and mdvi-1 treatment (Fig. 5 D). Finally, mRNA PPAR $\gamma$ expression, as an adipogenesis marker, was reduced in $o b / o b$ mice with and without leptin and mdvi-1 treatment compared to $w t$ mice (Fig. 5 E).

\section{Drp1 inhibition improves the metabolism in isolated adipocytes}

As leptin-deficient $o b / o b$ mice have a decreased oxidative metabolism of lipids and glucose (Lindström, 2007 ), we studied in vitro ${ }^{14}[\mathrm{C}]$ palmitic acid and D-glucose ${ }^{14}[\mathrm{C}]$ oxidation in isolated adipocytes incubated with leptin, mdvi-1 or siRNADrp-1 from wt C57BL/6 and ob/ob mice. Basal palmitic acid oxidation in adipocytes fromob/ob was $\sim 20 \%$ of the control group (Fig. 6 A). Leptin, mdvi-1 and siRNADrp-1 significantly increased palmitic acid oxidation $(3.8 \mathrm{pmol} / \mathrm{min} . / \mathrm{mg}$.protein, $3.3 \mathrm{pmol} / \mathrm{min} . / \mathrm{mg}$.protein, and 2.55 $\mathrm{pmol} / \mathrm{min} . / \mathrm{mg}$.protein, respectively) in $o b / o b$ compared toob/ob basal levels $(1.5 \mathrm{pmol} / \mathrm{min} . / \mathrm{mg}$.protein) (Fig. 6 A). Likewise, leptin, mdvi-1 and siRNADrp-1 treatment significantly increased glucose oxidation $(20 \mathrm{nmol} / \mathrm{min} . / \mathrm{mg}$.protein, $22 \mathrm{nmol} / \mathrm{min} . / \mathrm{mg}$.protein, and $26 \mathrm{nmol} / \mathrm{min} . / \mathrm{mg}$.protein, respectively) inob/ob compared to $o b / o b$ basal levels $(5.4 \mathrm{nmol} / \mathrm{min} . / \mathrm{mg}$.protein) (Fig $6 \mathrm{~B}$ ).

The activity of the mitochondrial electron transport chain (ETC) complex I was evaluated in isolated adipocytes mitochondria. As expected, an impairment of mitochondrial function was observed in $o b / o b(-50 \%)$, while leptin, mdvi-1 and siRNADrp-1 caused a 5 -fold increase (Fig. $6 \mathrm{C}$ ). No changes were found in $w t$ $C 57 B L / 6$ under any treatment.

\section{Effect of leptin and mdvi-1 in blood glucose level, body weight and food intake}

Measurements of blood glucose levels revealed that treatment with both leptin and mdvi-1 decreased the blood glucose concentration by $50 \%$ inob/ob mice almost to the $w t$ level (Fig. $7 \mathrm{~A}$ ). We did not observe any changes in body weight and food intake over the course of the treatments (Fig. 7 B and C).

\section{DISCUSSION}

We focused on mitochondrial dynamics and biogenesis as both processes are involved in WAT in obesity and type 2 diabetes. Mitochondria occupy an important position as mediators of cellular homeostasis, this position associates the dysfunction of mitochondria to the development of various human diseases.

Mitochondria are dynamic organelles, in a constant fusion and fission balance. Thus, mitochondrial networks respond to metabolic signals by increasing the actual mass of the network (biogenesis) as well as dispersing the existing mitochondria into a larger network (fission) (Nisoli et al.,2004). Drp1 is a master regulator of mitochondrial fission and fragmentation, while in contrast, Mfn2 and Opa1 promote mitochondrial fusion and branching. At the same time PGC- $1 \alpha$ promotes mitochondrial DNA replication (Zorzano et al., 2010).

Mitochondrial fission is a coordinated alternative response to an increase in metabolic load. In this study, we used leptin-deficientob/ob mice as a genetical model of obesity and diabetes and epididymal white adipose tissue as representative of visceral adipose tissue. Herein we report that mitochondrial fission and degradation 
are increased in these animals and that the administration of leptin normalized the Drp1, Mfn2 and Opa1 protein levels and reduced mitochondrial fragmentation.

Upon observing that Drp1 is overexpressed in WAT from $o b / o b$ mice, we decided to treat these mice with a pharmacological Drp1 inhibitor. Meaningfully, the effects of leptin administration were mimicked by the Drp1 inhibitor mdvi-1, which increased the percentage of tubular mitochondria and decreased the amount of fragmented mitochondria, disclosing that the defects observed in mitochondrial morphology are linked to Drp1 activation.

To confirm matching changes of mitochondrial morphology in cells, we studied the effect of leptin or mdvi1 in isolated adipocytes from treated mice, and we observed an increment of longer mitochondria, as a result of increased fusion and decreased fission. The stronger pro-fusion and anti-fission effect of leptin and mdvi-1 could explain the proportional increase of complex shaped mitochondria as observed in the "branched mitochondria" group. Elongated and branched mitochondria have been previously associated with improved OXPHOS and several bioenergetic advantages, while round mitochondria have been associated with a predominance of mitochondrial fission, increased mitophagy and poor bioenergetic performance (Chan, 2012; Scarpulla et al., 2012).

There is abundant literature showing that AMPK activates mitochondrial biogenesis and oxidation and AMPK-p, which is now considered a guardian of metabolism and mitochondrial homeostasis, is expressed in mitochondria from normal adipocytes (Fernandez-Veledo et al., 2013). Adipocytes from visceral adipose tissue of obese release smaller amounts of adiponectin, which leads to reduced activation of the AMPK/PGC1 pathway in tissues (Guilherme et al., 2008). We observed thatob/ob is an obesity and type 2 diabetes animal model with AMPK-p deficiency in WAT, but its expression can be restored with leptin or mdvi-1 treatment.

Mitochondrial biogenesis attenuates oxidative stress by enhancing mitochondrial capacity to manage metabolic reducing equivalents while increasing mitochondrial mass, and mitochondrial fission increases the number of individual mitochondria (Calabrese et al., 2012). Under basal conditions, constitutive PGC- $1 \alpha$ and Mfn2 expression controls the extent of mitochondrial biogenesis and regulates mitochondrial metabolism (Scarpulla, 2011). Accordingly, both proteins are induced when enhanced energy expenditure is required stimulating mitochondrial biogenesis and function. Evidence supports alterations in mitochondrial dynamics in insulin-resistance states both in humans and rats, and reduced expression of PGC-1 $\alpha$ and Mfn2 has been reported in obesity and type 2 diabetes (Martin et sl., 2014). Herein, we demonstrate for the first time that coincidentally leptin and mdvi-1 increase mitochondrial biogenesis and mass in $o b / o b$ mice.

Brown adipocytes are able to oxidize their own fat stores and circulating substrates, therefore producing heat and increasing metabolic rate. It has been shown that UCP-1 positive cells in WAT depict very similar properties as BAT cells (Herz et al., 2019). Thus, the highest capacity for "browning" or "beiging", and the action of browning WAT into BAT-like adipocytes in subcutaneous WAT in mice has been further postulated to protect against obesity. Similar to BAT adipocytes, beige adipocytes have the ability to take on a BAT-like thermogenic phenotype in response to various stimuli such as cold, endocrine factors or chemical compounds. Brown and beige adipocytes have a high level of mitochondrial content, contributing to high metabolic rates in these cells (Barbatelli et 1.,2010).

In the studied area we observed a decreased density of adipocytes, increased vascularization, which is representative of the browning process, and reduced adiposity in white adipose tissue from $o b / o b$ mice treated with leptin or mdvi-1.

In addition, mRNA UCP-1 was only increased bytheDrp1 pharmacological inhibitor while no changes were detected with leptin treatment. In our investigation AMPK phosphorylation in $o b / o b$ adipose tissue homogenates revealed that AMPK was completely deficient in its phosphorylated form (active). Our findings are in agreement with the loss of AMPK-p in WAT in obesity and diabetes, which is likely a driving force for re-whitening (inactivation) of beige adipocytes, exacerbating insulin resistance through impaired brown and beige fat function (Kaisanlahti et al.,2019). 
Leptin production by adipocytes is critical for energy homeostasis and body weight control, being the promotion of BAT thermogenesis, one of the mechanisms by which leptin increases energy expenditure. Leptin action in the hypothalamus increases sympathetic nerve activity to BAT, incrementing UCP-1 expression. UCP-1-mediated thermogenesis is a hallmark of brown/beige adipocytes development, albeit UCP1independent thermogenesis also occurs (Choe et al., 2016). Thus, the reduced adiposity that we observed with short leptin treatment could be independent of UCP-1. Differentiation and proliferation of adipocytesin vivo require PPAR $\gamma$ which is a ligand-activated transcription factor in the form of a nuclear receptor. It is highly expressed in adipose tissue, where it mostly regulates adipogenesis, lipid metabolism, and energy metabolism. Likewise, it also increases glucose utilization, promotes the metabolic action of insulin, collaterally increases adiponectin production, and shows a beneficial inhibitory effect on inflammatory processes. Furthermore, obesity has been associated with lower PPAR $\gamma$ expression. The expression of the different PPAR $\gamma$ isoforms may have dissimilar functions, for example PPAR $\gamma 2$, a nutrition regulated isoform is higher than PPAR $\gamma 1$ in obese individuals and enhance adipogenesis. Additionally, low calorie food can down-regulate the expression of PPAR $\gamma 2$ in adipose tissue. In contrast, PPAR $\gamma 1$ expression did not show a similar effect (Wang et al.,2017). PPAR $\gamma$ mRNA is reduced in $o b / o b$ mice and its expression is not increased with leptin or mdvi-1 treatment.

Mitochondria are the major sites of cellular ROS production, but are also sensitive to damage by ROS. Obesity and insulin resistance has been involved in oxidative stress, particularly focused on the complex I activity, (Kusminski et al., 2012). Adipose, skeletal muscle and liver are major organs involved in glucose and free fatty acid (FFA) metabolism and play an important role in insulin resistance. The FFA are mobilized from adipose tissue and ectopically accumulated in liver, skeletal muscle and $\beta$ cells resulting in dysregulated glucose metabolism, lipid-induced insulin resistance, inflammation, increased ROS production and decreased PGC-1 $\alpha$ expression (Blake et al.,2014). As a reciprocal influence between bioenergetics and mitochondrial dynamics, diabetic neurons initially respond to hyperglycemia by increasing mitochondrial biogenesis and fission but, over time and with accumulation of ROS, the balance leans toward mitochondrial fission, producing small aberrant mitochondria with reduced respiratory capacity. Thus, mitochondrial respiration is profoundly influenced by mitochondrial dynamics (Ren et al., 2010).

In previous studies, we have shown that the presence of hyperinsulinemia stimulates the production of NO, the nitration of mitochondrial complex I, mitochondrial dysfunction and a reduction in oxidative metabolism in adipose tissue (Finocchietto et al., 2008; Finocchietto et al., 2011). In the present study, we detected mitochondrial dysfunction led by a decreased complex I activity and oxidative metabolism in ob/obadipocytes, counteracted by leptin, mdvi-1 and siRNAs Drp-1, which increased complex I activity. It was followed by an increment of glucose and palmitic acid oxidation, depicting that mitochondrial function is closely related to mitochondrial homeostasis.

In accordance with the metabolic and mitochondrial effects, leptin and mdvi-1 treatments produced a significant decrease in blood glucose levels in $o b / o b$ mice which typically exhibit high levels of blood glucose concentration. Normalization of blood glucose levels were not accompanied by a decrease in body weight and food intake, suggesting that the blood glucose concentration was related to increased glucose oxidative metabolism secondary to changes in mitochondrial homeostasis.

In summary, mitochondrial dysfunction has been related with obesity and type 2 diabetes, two close conditions recently named as diabesity, and considered a modern epidemic. $O b / o b$ mice is a model of leptin deficiency with hyperinsulinemia that induce mitochondrial dysfunction due to increased oxidative and nitrosative stress, while AMPK-p is reduced. Abnormal mitochondrial morphology and impaired dynamics are an important determinant of the mitochondrial dysfunction affecting respiratory. The inhibition of mitochondrial fission by mdvi-1 or leptin stimulates mitochondrial biogenesis, fusion and oxidative capacity. Drp1, a critical mediator of mitochondrial fission, also has an important role in browning white adipose tissue into beige adipocytes.

We suggest that mitochondrial fission inhibition through Drp-1 blocking can be now proposed as a novel therapeutic target for obesity and diabetes. Based on that, it seems reasonable to predict that future new 
therapies targeting mitochondrial fission, as mdvi-1 or better pharmacological agents, could be tested as potential alternatives.

\section{Acknowledgments}

The authors thank Margarita López, Fabiana Confente and Mariana López Ravasio for electron microphotographs, Inés Rebagliatta and Silvia Holod for technical support; and Natalia Riobó for revising of the manuscript.

\section{Funding}

This work was supported by the Agencia Nacional de Promoción Científica y

Tecnologica (FONCyT) grant, PICT 1781 and Universidad de Buenos Aires grant, UBACyT 20020130100574BA (to M.C.C.).

Duality of Interest. No potential conflicts of interest relevant to this article were reported.

\section{Author Contribution}

P.F., M.C.C., and J.J.P. designed the study; P.F. performed experiments; H.P. performed the RNA extraction, Quantitative Real-Time PCR, and mtDNA content experiment; G.B performed fluorescence microscope experiment, V.M and G.B performed optic microscope and area, density and vascular white adipose tissue study, C.M performed technical assistant, C.M performed histological study, P.F., and M.C.C. collected and analyzed data; P.F., J.P, J.J.P and M.C.C. wrote the manuscript.

\section{REFERENCES}

1-Leitner DR, Fruhbeck G, Yumuk V, Schindler K, Micic D, Woodward E, and Toplak H. (2017). Obesity and Type 2 Diabetes: Two Diseases with a Need for Combined Treatment Strategies - EASO Can Lead the Way. Obes Facts. 10(5):483-492.

2-Boden G (2011). Obesity, insulin resistance and free fatty acids. Curr. Opin. Endocrinol. Diabetes Obes. 18:139-143.

3-Hildebran S, Stumer J, Pfeifer A (2018). PVAT and its relation to brown, beige and white adipose tissue in development and function. Front. Physiol. 9:70.doi: 10.3389/fphys.2018.00070.

4-Pfeifer A, and Hoffmann, LS (2015). Brown, beige,and white:the new color code of fat and its pharmacological implications. Annu. Rev. Pharmacol. Toxicol. 55, 207-227. doi: 10.1146/annurev-pharmtox010814-124346.

5-ChenY, Pan R, Pfeifer A (2017). Regulation of brown and beige fat by microRNAs. Pharmacol. Ther. 170, 1-7. doi: 10.1016/j.pharmthera.2016.10.00.

6-Chandel NS (2014). Mitochondria as signaling organelles. BMC Biol.12:34-42.

7-Kotiadis VN, Duchen MR, and Osellame LD (2014). Mitochondrial quality control and communications with the nucleus are important in maintaining mitochondrial function and cell health. Biochim. Biophys. Acta. 1840:1254-65.

8-Westermann B. Mitochondrial fusion and fission in cell life and death (2010). Nature Rev Mol Cell Biol. 11: $872-84$.

9- Mootha Youle RJ, and Narendra DP (2011). Mechanisms of mitophagy. Nat. Rev. Mol. Cell Biol. 12:9-14.

10-Chen H and Chan DC (2005). Emerging functions of mammalian mitochondrial fusion and fission. Hum Mol Genet 14: R283-R289.

11-Yu T, Robotham JL, Yoon Y (2006). Increased production of reactive oxygen 
species in hyperglycemic conditions requires dynamic change of mitochondrial

morphology.Proc Natl Acad Sci U S A.103: 2653-2658.

12- Smith G and Gallo G (2017).To mdivi-1 or not to mdivi-1: Is that the question?

Dev Neurobiol.77(11): 1260-68.

13- Whitaker RM, Corum D, Beeson CC, and Schnellmann RG (2016). Mitochondrial Biogenesis as a Pharmacological Target: A New Approach to Acute and Chronic Diseases. Annu Rev Pharmacol Toxicol.56:22949.

14- Desjardins EM \& Steinberg GR (2018). Emerging Role of AMPK in Brown and Beige Adipose Tissue (BAT): Implications for Obesity, Insulin Resistance, and Type 2 Diabetes. Current Diabetes Reports.18: 80.

15-Baker MJ, Palmer CS, and Stojanovski D (2014). Mitochondrial protein quality control in health and disease. Br. J. Pharmacol. 171:1870-89.

16-Archer SL (2013). Mechanisms of Disease Mitochondrial Dynamics - Mitochondrial Fission and Fusion in Human Diseases. N Engl J Med.369:2236-51.

17- Perez,H Finocchietto,PV, Alippe Y, Rebagliati I, Elguero ME, Villalba N, Poderoso JJ, Carreras MC (2018). p66Shc Inactivation Modifies RNS Production, Regulates Sirt3 Activity, and Improves Mitochondrial Homeostasis, Delaying the Aging Process in Mouse Brain Oxidative Medicine and Cellular Longevity113.https://doi.org/10.1155/2018/8561892.

18- Gonzalez AS, Elguero ME,Finocchietto P, Holod S, Romorini L, Miriuka SG, Peralta JG, Poderoso JJ,Carreras MC (2014).Abnormal mitochondrial fusion - fi ssion balance contributes to the progression of experimental sepsis. Free Radical Research.1-15. ISSN 1071-5762 print/ISSN 1029-2470 online DOI: 10.3109/10715762.2014.906592

19- Rovira-Llopis S, Banulsa C, Diaz-Moralesa N, Hernandez-Mijaresa A, Milagros Rochaac M, Victor M (2017). Changes in shape or size of mitochondria have been observed in diabetic patients and animal models (Mitochondrial dynamics in type 2 diabetes: Pathophysiological implications. Redox Biology.11:637-645.

20-Finocchietto P, Barreyro F, Holod S, Peralta J, Franco MC, Mendez C, Converso DP, Estevez A, Carreras MC, Poderoso JJ (2008). Control of Muscle Mitochondria by Insulin Entails Activation of Akt2-mtNOS Pathway: Implications for the Metabolic Syndrome. PLoS ONE. 3(3):e1749.

21-Finocchietto P, Holod S, Barreyro F, Peralta JG, Alippe Y, Giovambattista G, Carreras MC, Poderoso JJ (2011). Defective Leptin-AMPK Pathway Induces NO Release and Causes Mitochondrial Dysfunction and Obesity in ob/ob Mice. Antioxidants \& Redox Signaling. 15: 2395-406.

22-Pelleymounter MA, Cullen MJ, Baker MB, Hecht R, Winter D, Boone T, Collins F (1995). Effects of the obese Gene Product on Body Weight Regulation in ob/ob Mice. Science. 269:540-543.

23- Ceddia, R.B (2005). Direct metabolic regulation in skeletal muscle and fat tissue by leptin: implications for glucose and fatty acids homeostasis. International Journal of Obesity. 29: 1175-1183.

24- Carpenter AE, Jones TR, Lamprecht MR, Clarke C, Kang IH, Friman O, et al. (2006). CellProfiler: image analysis software for identifying and quantifying cell phenotypes. Genome biology.7 (10): R100.

25- Jones TR, Kang IH, Wheeler DB, Lindquist RA, Papallo A, Sabatini DM, et al. . (2008).CellProfiler Analyst: data exploration and analysis software for complex image-based screens. BMC bioinformatics.9: 482.

26- Jones TR, Carpenter AE, Lamprecht MR, Moffat J, Silver SJ, Grenier JK, et al. (2009).Scoring diverse cellular morphologies in image-based screens with iterative feedback and machine learning. Proceedings of the National Academy of Sciences of the United States of America. 106(6): 1826-1831. 
27-Venegas V, Wang J, Dimmock D, Wong LJ (2011). Real-Time Quantitative PCR Analysis of Mitochondrial DNA Content.Curr. Protoc. Hum. Genet. 68:19.7.1-19.7.12.

28- Otera H, Shihara N, K Mihara K (2013). New insights into the function and regulation of mitochondrial fission. Biochimica et Biophysica Acte. 5 :1256-1268.

29-Twig G, Shirihai OS (2011). The Interplay Between Mitochondrial Dynamics and Mitophagy. Antioxidants \& Redox Signaling. 14, DOI: 10.1089/ars.2010.3779.

30-Navarro-Yepes J, Burns M, Anandhan A, Khalimonchuk O, del Razo LM, Quintanilla-Vega B, Pappa A, Panayiotidis MI, and Franco R (2014). Oxidative Stress, Redox Signaling, and Autophagy: Cell Death Versus Survival. Antioxidants \& Redox Signaling. DOI: 10.1089/ars.2014.5837.

31-Galloway CA, Lee H, Yoon Y (2012). Mitochondrial morphology-emerging role in bioenergetics. Free radical biology \& medicine. 53(12): 2218-2228.

32-Jeon SM (2016). Regulation and function of AMPK in physiology and diseases. Experimental \& Molecular Medicine. 48, e245; doi:10.1038/emm.2016.81.

33- Jager S, Handschin C, St-Pierre J, Spiegelman BM (2007). AMP-activated protein kinase (AMPK) action in skeletal muscle via direct phosphorylation of PGC-1alpha. Proc. Natl. Acad. Sci. USA.104:12017-12022.

34- Zorzano A, Liesa M, and Palacin M (2009). Role of mitochondrial dynamics proteins in the pathophysiology of obesity and type 2 diabetes. Int J Biochem Cell Biol. 41: 1846-54.

35- Liangyou Rui (2018). Brown and Beige Adipose Tissues in health and Disease. Compr Physiol.7(4): $1281-1306$.

36- Lindstrom P (2007). The Physiology of Obese-Hyperglycemic Mice [ob/ob Mice]. The Scientific World Journal. 7, 666-685 ISSN 1537-744X; DOI 10.1100/tsw.2007.117.37- Nisoli E, Clementi E, r Moncada S, and Carruba MO (2004). Mitochondrial biogenesis as a cellular signaling framework. Biochemical Pharmacology. $67: 1-15$.

38-Zorzano A, Liesa M, Sebastian D, Segales J, and Palacin M (2010). Mitochondrial fusion proteins: dual regulators of morphology and metabolism. Semin Cell Dev Biol. 21: 566-74.

39-Chan DC (2012). Fusion and fission: interlinked processes critical for mitochondrial health. Annu Rev Genet. 46: 265-287.

40-Scarpulla RC, Vega RB, and Kelly DP (2012). Transcriptional integration of mitochondrial biogenesis. Trends Endocrinol. Metab. 23:459-66.

41- Fernandez-Veledo S, azquez-Carballo A, ila-Bedmar R, Ceperuelo Mallafre V, Vendrell J (2013). Role of Energy- and Nutrient-sensing Kinases AMP-activated protein kinase (AMPK) and mammalian target of rapamycin (mTOR) in Adipocyte Differentiation.International Union of Biochemistry and Molecular Biology, IUBMB Life. DOI: 10.1002/iub.1170.

42- Guilherme A, Virbasius JV, Puri V, Czech MP (2008). Adipocyte dysfunctions linking obesity to insulin resistance and type 2 diabetes. Nat. Rev. Mol. Cell Biol. 9:367-377.

43-Calabrese V, Cornelius C, Leso V, Trovato-Salinaro A, Ventimiglia B. et al (2012). Oxidative stress, glutathione status, sirtuin and cellular stress response in type 2 diabetes. Biochim. Biophys. Acta. 1822:72936.

44-Scarpulla RC (2011). Metabolic control of mitochondrial biogenesis through the

PGC-1 family regulatory network. Biochim Biophys Acta. July ; 1813(7): 1269-1278

45- Martin SD, McGee SL (2014). The role of mitochondria in the aetiology of insulin resistance and type 2 diabetes. Biochim. Biophys. Acta. 1840:1303-12. 
46- Herz CT and Kiefer FW (2019). Adipose tissue browning in mice and humans.

Journal of Endocrinology. 241, R97-R109.

47- Barbatelli G, Murano I, Madsen L, Hao Q, Jimenez M, Kristiansen K, Giacobino JP, De Matteis R \& Cinti S (2010). The emergence of cold induced brown adipocytes in mouse white fat depots is determined predominantly by white to brown adipocyte transdifferentiation. American Journal of Physiology: Endocrinology and Metabolism. 298 E1244-E1253. https://doi.org/10.1152/ajpendo.00600.2009.

48-Kaisanlahti A \& Glumoff T (2019). Browning of white fat: agents and implications for beige adipose tissue to type 2 diabetes. Journal of Physiology and Biochemistry 75:1-10.

49- Choe SS, Huh, JY , Hwang IJ, Kim JI, Kim JB (2016). Adipose Tissue Remodeling: Its Role in Energy Metabolism and Metabolic Disorders. Front. Endocrinol. 7,30:1-6.

50- Wang Q,Imam MU, Zhang Yida Z, Wang F (2017).Peroxisome Proliferator-Activated Receptor Gamma (PPAR $\gamma$ ) as a Target for Concurrent Management of Diabetes and Obesity-Related Cancer. Current Pharmaceutical Design. 23, 3677-3688.

51-Kusminski, C. M., and Scherer, P. E (2012). Mitochondrial dysfunction in white adipose tissue. Trends Endocrinol. Metab. 23,435-443.

52-Blake R, and Trounce IA (2014). Mitochondrial dysfunction and complications associated with diabetes. Biochim. Biophys. Acta. 1840:1404-12.

53-Ren J, Pulakat L, haley-Connell A, Sowers JR (2010). Mitochondrial biogenesis in the metabolic syndrome and cardiovascular disease. J Mol Med. 88:993-1001.

\section{LEGENDS of the FIGURES}

\section{Figure 1- Mitochondrial dynamics in WAT}

(A) Representative immunoblot of proteins separated using SDS-PAGE from whole WAT lysate reveals the expression of the fission protein Drp1, (B) active isoform of Drp-1 phosphorylated in Ser 616, (C) inactive isoform of Drp-1 phosphorylated in Ser 637, and (D and E) the expression of fusion proteins Mfn2 and Opa-1. Actin protein expression was used as standard. Bars reflect the densitometry in arbitrary units (A.U.) in absolute amount of protein. ${ }^{*} \mathrm{p}<0.05$ denotes different from respective $w t C 57 B L / 6$. ${ }^{* *}$ denotes different from respective $o b / o b,{ }^{* *} \mathrm{p}<0.05 o b / o b$ vs $o b / o b+$ leptin treatment $(1 \mathrm{mg} / \mathrm{kg}$ i.p). n:[?] 6-7 per group. One-way analysis of variance (ANOVA) and Bonferroni post hoc test.

\section{Figure 2-Adipose mitochondrial ultrastructural morphology}

Representative electron microscope images of mitochondrial morphology were evaluated using electron microscopy of fixed white adipose tissue from wt C57BL/6 (A) or $o b / o b$ (B) (Magnification 3000-30.000 X) with leptin (C), or mdvi-1 (D) treatments (bar represents = micrometer), (5 images per animal for [?] 6 per group). Tubular and fragmented mitochondria were counted per arbitrary area.

(E) The percentage distribution of tubular and fragmented adipocyte mitochondria was determined in a minimum of 8-10 random fields at 3.000-30.000 X magnification to ensure a representative area of analysis. Those mitochondria whose length were more than thrice its width were considered tubular while round mitochondria were considered fragmented.

(F) Representative images of WAT $o b / o b$ mice slices reveal the presence of mitophagy characterized by autophagic vacuoles with mitochondrial particles or mitophagosomes (black arrows) (total images of mitophagy in 10 images per animals).

Figure 3- Mitochondria shape-classes by leptin or mdvi-1 treatment Mitochondrial segmentation and measurement of morphological parameters followed by supervised classification into three mitochondrial shape-classes (to further determine proportions of each class). 
(A) Representative micrographs of adipocytes stained with mitotracker green and observed under fluorescence microscopy (digitally enlarged $1.000 \mathrm{x}$ magnification), and their matching segmentation obtained with the Cell Profiler pipeline for extracting morphological measurements.

(B) Proportions of the three defined mitochondria shape-classes among treatment groups with their corresponding $95 \%$ confidence intervals, as obtained from scoring with supervised classification.

Figure 4- Effect of leptin or mdvi-1 on AMPK and PGC-1 $\alpha$ in WAT

(A) Representative immunoblot of proteins separated using SDS-PAGE from whole WAT lysate reveals the expression of AMPK and AMPK-P in $w t, o b / o b, o b / o b+$ leptin or mdvi-1. Actin protein expression was used as standard. (B) Bars reflect the protein densitometry in arbitrary units (A.U.). n. ${ }^{*} \mathrm{p}<.0 .5$ vs wt C57BL/6

. ${ }^{* *}$ denotes different from respective $o b / o b . \mathrm{p}<0.05$ versus $w t C 57 B L / 6$ vs $o b / o b{ }^{* *} \mathrm{p}<0.05 o b / o b$ vs $o b$ + leptin or mdvi-1 n: 5-7 per group. One-way analysis of variance (ANOVA) and Bonferroni post hoc test.

(C) The mRNA levels of PGC-1 $\alpha$ were measured in TRIZOL-treated WAT extracts from mice treated with leptin or mdvi-.

(D) Quantification of mtDNA and nuclear DNA content by qPCR in adipose tissue in $w t C 57 B L / 6, o b / o b$ and $o b / o b+$ leptin or mdvi-1 treatment, mtDNA/nDNA ratio was calculated. Results were expressed as the mean \pm SEM, n [?] 6, per duplicate and $* \mathrm{p}<0.05$ wt C57BL/6 vs ob/ob, ** $\mathrm{p}<0.05 o b / o b$ vs ob/ob + leptin or mdvi-1, n: 5-7 per group. One-way analysis of variance (ANOVA) and Bonferroni post hoc test.

Figure 5- Brown-like adipose tissue and UCP1 expression with leptin or mdvi-1 treatment

(A) Representative micro-sections of WAT were stained with hematoxylin and eosin reagent and periodic acid-schiff (PAS) stain for size determination (B) and vascular density adipocyte (C) in all studied groups. (D) The mRNA levels of UCP1 and (E) PPAR $\curlyvee$ was measured in Trizol-treated WAT extracts from all the studied groups; GADPH mRNA was used as standard ( $\mathrm{n}>6)$. ${ }^{*} \mathrm{p}<0.05$ wtC57BL/6 vs ob/ob, ${ }^{* *} \mathrm{p}<$ $0.05 \mathrm{ob} / \mathrm{ob} \mathrm{vsob} / \mathrm{ob}+$ leptin or mdvi-1. One-way analysis of variance (ANOVA) and Bonferroni post hoc test.

\section{Figure 6- Metabolic effects of leptin or mdvi-1 on adipocytes}

(A) Adipocytes from $w t$ and $o b / o b$ mice were isolated and incubated with leptin, mdvi-1 or siRNA DRP1 and fat metabolism was measured as fatty acid oxidation with $1 \mu \mathrm{Ci} / \mathrm{ml}^{14}[\mathrm{C}]$-palmitic acid oxidation to $\mathrm{CO}_{2}$ and $\mathrm{H}_{2} \mathrm{O}$. Data resulted from pmolar/min./mg protein (Eli Wallac, $; \mathrm{n}=5-6$ per triplicate). (B) Glucose oxidation was measured with $1 \mu \mathrm{Ci} / \mathrm{ml}^{14}[\mathrm{C}]$ - complete glucose oxidation to $\mathrm{CO}_{2}$ and $\mathrm{H}_{2} \mathrm{O}$. Data resulted from nmolar $/ \mathrm{min} . / \mathrm{mg}$ protein (Eli Wallac, $\mathrm{n}=5-6$ per triplicate). (C) Mitochondrial complex I activity was measured following the reduction of cytochrome $\mathrm{c}$ at $30^{\circ} \mathrm{C}$ in the presence of NADH and KCN. The reaction rate was measured as the pseudo - first-order reaction constant ( $\left.\mathrm{k}^{\prime}\right)$ and expressed as k' $/ \mathrm{min}$. mg protein. test.

Figure 7- Effect of leptin or mdvi-1 on blood glucose level, body weight and food intake

(A) Blood glucose concentration (mg/dl) was measured in all studied groups. (B) Body weight (g) and (C) food intake $(\mathrm{g} / \mathrm{d})$ were determined in all animals during the study. Results are expressed as mean \pm SEM for $6-8$ rats per duplicate, and ${ }^{*} \mathrm{p}<.0 .5$ vs wtC57BL/6. ${ }^{* *}$ denotes different from respective $o b / o b . \mathrm{p}<$ 0.05 versus $w t C 57 B L / 6$ vs $o b / o b^{* *} \mathrm{p}<0.05 o b / o b$ vs $o b+$ leptin or mdvi-1 n: 5-7 per group. One-way analysis of variance (ANOVA) and Bonferroni post hoc

\section{SUPPLEMENTAL MATERIAL}

Supplemental Table I . Mice gene-specific oligonucleotide sequences used in quantitative real-time polymerase chain reaction. 


\begin{tabular}{|c|c|c|}
\hline Gene & $\begin{array}{l}\text { Primer sequence ( } 5^{\prime} \text { to } \\
\left.3^{\prime}\right)\end{array}$ & $\begin{array}{l}\text { Annealing Temperature } \\
{ }^{\circ} \mathrm{C}\end{array}$ \\
\hline$\overline{P G C 1-\alpha \mathrm{Fw}}$ & GACCCTCCTCACACCAAACOCC & 60 \\
\hline PGC1- $\alpha$ Rv & TTTGGTGACTCTGGGGTCAGAG & \\
\hline $\mathrm{UCP}-1 \mathrm{Fw}$ & AGGCTTCCAGTACCATTA@GT & 60 \\
\hline $\mathrm{UCP}-1 \mathrm{Rv}$ & CTGAGTGAGGCAAAGCTGATTT & \\
\hline GAPDH Fw & AGACAGCCGCATCTTCTT\&T & 62 \\
\hline GAPDH Rv & TGATGGCAACAATGTCCACT & \\
\hline mtDNA $16 \mathrm{~S}$ rRNA Fr & GCCTTCCCCCGTAAATGЯTA & 62 \\
\hline mtDNA $16 \mathrm{~S}$ rRNA R & TTATGCGATTACCGGGCTCT & \\
\hline $\mathrm{nDNA} ß 2 \mathrm{M} F \mathrm{w}$ & TGCTGTCTCCATGTTTG & 62 \\
\hline nDNA ß2M Rev & TCTCTGCTCCCCACCTCTAAGT & \\
\hline
\end{tabular}

GADPDH, glyceraldehyde 3-phosphate dehydrogenase;

ß2M, $\beta 2$-microglobulin

FW, forward; RV, reverse.

\section{Supplemental Figure 1: Assessment of the fraction of mitochondria shape-classes by supervised classification}

A Cell Profiler pipeline was created for the segmentation and measurement of morphometric parameters of individual mitochondria. Several images were processed with this pipeline for each treatment and morphometric parameters were exported and processed with Cell Profiler Analyst (Broad Institute, USA). A classification algorithm was used to implement a machine learning approach to classify mitochondria intro three groups: round, elongated and branched. The training procedure was repeated until global classification accuracy achieved $90 \%$, with $100 \%$ classification accuracy of the round mitochondria subgroup (further details a references in methods section).

(A) An example of a supervised classification of a single image used to verify the ability to discriminate among morphological classes.

(B) A sample of the training set used for machine learning and the confusion matrix obtained after training. The matrix was used to confirm the accuracy of the scoring of the whole set of mitochondria.

\section{Hosted file}

FIGURES bis.pptx available at https://authorea.com/users/346499/articles/472469-inhibitionof-mitochondrial-fission-by-drp-1-blockade-improves-white-adipose-tissue-abnormalitiesin-obesity-and-diabetes 